\title{
Asymmetric Uncertainty Expression for High Gradient Aerodynamics
}

\author{
Jeremy T. Pinier* \\ NASA Langley Research Center, Hampton, VA, 23681-2199
}

\begin{abstract}
When the physics of the flow around an aircraft changes very abruptly either in time or space (e.g., flow separation/reattachment, boundary layer transition, unsteadiness, shocks, etc), the measurements that are performed in a simulated environment like a wind tunnel test or a computational simulation will most likely incorrectly predict the exact location of where (or when) the change in physics happens. There are many reasons for this, including the error introduced by simulating a real system at a smaller scale and at non-ideal conditions, or the error due to turbulence models in a computational simulation. The uncertainty analysis principles that have been developed and are being implemented today do not fully account for uncertainty in the knowledge of the location of abrupt physics changes or sharp gradients, leading to a potentially underestimated uncertainty in those areas. To address this problem, a new asymmetric aerodynamic uncertainty expression containing an extra term to account for a phase-uncertainty, the magnitude of which is emphasized in the high-gradient aerodynamic regions is proposed in this paper. Additionally, based on previous work, a method for dispersing aerodynamic data within asymmetric uncertainty bounds in a more realistic way has been developed for use within Monte Carlo-type analyses.
\end{abstract}

\section{Nomenclature}

\section{Symbols}

$\begin{array}{ll}C_{i} & =\text { aerodynamic coefficient } \\ a_{k}, b_{k}, h_{k} & =\text { randomly generated coefficients } \\ \epsilon_{i} & =\text { randomly generated dispersion factor } \\ f & =\text { functional } \\ \widetilde{f} & =\text { dispersed functional } \\ g_{i} & =\text { randomly generated dispersion function } \\ L_{b} & =\text { bias limit } \\ M & =\text { Mach number } \\ N & =\text { order of the partial Fourier series } \\ U & =\text { uncertainty magnitude } \\ u^{-} & =\text {lower uncertainty bound } \\ u^{+} & =\text {upper uncertainty bound } \\ \Delta u^{-} & =\text {lower uncertainty increment } \\ \Delta u^{+} & =\text {upper uncertainty increment } \\ L / D & =\text { lift-to-drag ratio } \\ \alpha & =\text { angle of attack }\end{array}$

* Research Aerospace Engineer, Configuration Aerodynamics Branch, MS 499, Senior Member AIAA. 


\section{Introduction}

Reducing uncertainty in aerodynamic data goes hand-in-hand with increasing one's confidence that the physics of the flow are well understood and realistically captured by a dataset and an uncertainty model. Deriving an uncertainty model for a vehicle's aerodynamic environment requires meticulous care to ensure that it captures all potential sources of error and addresses any 'lack of knowledge' type of uncertainty, while remaining a realistic representation of the aerodynamics of the vehicle. Statistics are traditionally used to provide the engineer with objective tools that capture the variability of a given measurement, and determine with a reasonable level of confidence a measurement uncertainty. However this variability is limited to what can be simulated in a controlled environment like a wind tunnel at scaled sizes. Full scale flight data will, in many cases, show variations from expected values beyond what has been estimated by measurement statistics and engineering judgment only, because of potentially misrepresented physics in the predicted pre-flight aerodynamic model. It is therefore important to understand the limitations of purely statistical approaches to uncertainty quantification to reduce the risks associated with an unrealistic predictive model. Moreover, statistics only provide a single-point type uncertainty expression, as opposed to a distributed expression. A single-point uncertainty in an output variable is expressed as a function of a single value of an input, whereas a distributed uncertainty in an output variable is expressed as a function of multiple input values, which allows for spatial or temporal correlation to be maintained in the uncertainty and dispersion models.

For demonstration purposes, and in an effort to apply it to existing experimental data, the new uncertainty expression proposed in this paper is applied to Apollo command module wind tunnel data from Moseley et al. ${ }^{1}$ Many wind tunnels around the country were used to characterize the aerodynamic environment of the Apollo command module, as explained in the aforementioned report. The particular subset of data that was extracted for use in this work was acquired at the Ames $2 \times 2$ foot Transonic Wind Tunnel. As shown in the present work this data exhibits large gradients due to flow separation and reattachment, as the command module is pitched from low angles of attack (apex forward) to high angles of attack (heat shield forward). The relevance of this type of aerodynamic data is very much in line with that of blunt-shaped vehicles currently being developed at NASA and in the industry, including the Orion Multi-Purpose Crew Vehicle (MPCV) capsule, and launch abort systems like the Max Launch Abort System (MLAS). Analysis of MLAS, MPCV, and Apollo aerodynamic data has shown that these vehicles present the same type of general aerodynamic characteristics with massively separated flow on the heat shield at low angles of attack $\left(0-30^{\circ}\right)$ and curved surface flow separation on the leeward side of the crew module area along with flow reattachment on the heat shield at high angles of attack $\left(50-90^{\circ}\right)$. This new type of uncertainty expression will increase the fidelity of the uncertainty models in high-gradient regions where aerodynamic stability could be an issue. This in turn, could reduce risks linked to misrepresented aerodynamic environments in flight simulations.

\section{High-gradient Aerodynamics and Physical Boundary Uncertainties}

This section describes how a vehicle can be subject to a range of flow phenomena and how understanding and accurately determining the boundaries of each flow domain can be challenging. Quantifying an uncertainty in the domain boundaries and propagating this uncertainty in a model such as the one proposed in this paper can provide the engineer more confidence that any lack of understanding of the physics be captured by appropriate uncertainty bounds and a physics-based dispersion method.

\section{A. High-gradient Aerodynamics}

In the context of this paper, the term high-gradient aerodynamics is used to describe the abrupt change of any flow quantity or integrated aerodynamic quantity in time or space. Flow quantities (like pressure, Mach number, velocity or density) and integrated aerodynamic loads on a vehicle usually exhibit smooth trends when the vehicle is operating within the boundaries of a physical flow state. As the vehicle changes attitude with respect to the freestream flow, or if the flow conditions vary, the overall flow around the vehicle could exhibit drastically different features as it suddenly evolves to a different physical state. The transition to this different state will manifest itself as a high gradient in data trends. Capturing the location (in time or space) of this transition in a predictive sense can prove to be crucial for ensuring that the vehicle can 
be controlled at all times in the flight simulations. In wind tunnel environments, or when computing flow properties with computational fluid dynamics (CFD), it is not guaranteed that the model faithfully represents what would be seen on the full scale flight vehicle in realistic conditions. The challenge is therefore to estimate the potential amount of error that could be introduced by the modeling and devise a method to express an uncertainty not only in magnitude but also in phase. When the gradients in the data are small, modeling a phase error is not as crucial since a single-point, symmetric type uncertainty model would, in most cases, be sufficient to allow for a small error in phase in the nominal data. However, in high gradient areas, traditional symmetric uncertainties fall short of modeling a sufficient phase uncertainty and, in the limit of an infinite gradient (e.g., a step function), such models imply perfect knowledge of the location of the step (i.e., the abrupt physical transition location). A model is proposed in the following section to allow for a phase error due to imprecise knowledge of the physical transition.

There are many instances where sharp gradients can be observed in the aerodynamic characteristics of atmospheric flight vehicles. Following are examples of physical phenomena that are most commonly at the origin of such gradients in aerodynamic flows:

- Flow separation and/or reattachment regions are usually very dynamic and unsteady environments, which poses a great challenge in predicting the exact conditions that trigger flow separation or reattachment. Small changes in vehicle attitude or flow conditions can have a dramatic effect on the state of the flow. Curved smooth surfaces in particular will exhibit multiple types of flow separation depending on the state of the boundary layer (laminar, turbulent) and the type of pressure gradient it is subject to. Polhamus ${ }^{2}$ and Luckring ${ }^{3}$ have provided insight into understanding how the physics of flows over wings is greatly sensitive to Reynolds number, Mach number, compressibility, leading edge radius, wing sweep and slenderness, among other configuration parameters. Different types of flow separation will trigger different types of wing aerodynamics (e.g. stall, buffet, leading-edge vortex formation, etc), which splits the parameter space into distinct flow domains exhibiting vastly different flow physics. Very small changes in flow conditions could therefore induce a transition from one flow domain to another with large gradients in the aerodynamic loads. Hysteresis loops are also a common occurrence when flow separation is present, which leads to a solution that is direction dependent and, when the direction is unknown, an uncertainty in the location of the transition between attached and separated slow states.

- Shock wave/boundary layer interactions, at transonic and supersonic conditions, are also very dynamic in nature and therefore result in an uncertain position and impact of the shock on the vehicle. Depending on the configuration of the vehicle, the shock wave could be standing in a very unsteady part of the flow and therefore show dynamic fluctuations in streamwise position. These dynamic fluctuations should be attempted to be captured to assess a minimum amount of uncertainty of the physical location of the shock. All flow quantities also exhibit strong discontinuities through a shock wave which makes their prediction difficult when its position is largely unsteady. Smits \& Muck ${ }^{4}$ found that the interaction between a separating turbulent boundary layer and a shock wave over a $20^{\circ}$ turning angle exhibits such unsteadiness that the structure of the shock and resulting normal stresses show larger than expected random variations. A traditional uncertainty model in this case would not necessarily be able to capture such dynamics in the state of the flow.

- Boundary layer transition remains a difficult phenomenon to measure in a wind tunnel environment, or simulate using CFD. Both methods are complementary ways to predict the characteristics of the a full-scale vehicle's flight environment. Experimentally, for some vehicles like traditional transport configurations, fairly well established techniques have been developed to simulate the boundary layer transition in a way that is representative of the full scale vehicle. ${ }^{5,6}$ Additionally, in a few cryogenic facilities, flight Reynolds numbers can be obtained, in which case natural transition should occur in a very similar way than in full-scale flight. However, for complex geometries, blunt bodies or slender bodies of revolution that exhibit flow separation, the techniques implemented to trip the boundary layer are still very much at the research level and in most cases, it is difficult to know whether the boundary layer is effectively tripped and remains turbulent over the entire scaled vehicle. For launch vehicles, flight Reynolds numbers can still be several orders of magnitude higher than in the testing environment, leaving room for Reynolds number scaling error. Additionally, natural transition on a full scale vehicle will occur at various stations on the vehicle depending on factors such as freestream 
turbulence intensity, gusts, model attitude, kinematic viscosity of ambient air, surface finish roughness, protuberance flow interactions, etc. Computationally, predicting laminar/turbulent boundary layer transition is a yet harder task since it requires time dependent simulations that are able to model the instabilities in the boundary layer that trigger transition. Simulating boundary layer transition in CFD is the subject of much research. There can therefore be discrepancies in the nature of the boundary layer between the simulated vehicle and the full-scale vehicle, especially when flow separation is present.

- Bi-modal flow behaviors are a phenomenon that can be observed most commonly at transonic conditions $(0.7 \leq \mathrm{M} \leq 1.3)$ where, for example, the flow has to turn around a sharp corner and the interaction between the separating flow and the shock is observed to have two stable states at very close free stream Mach number conditions. An example of such a behavior has been reported by Piatak et al. ${ }^{7}$ resulting from rigid buffet wind tunnel testing performed on the Ares I-X flight test vehicle. Pinier \& Niskey ${ }^{8}$ have also reported a bi-modal type phenomenon in the stage separation aerodynamics of the Ares I-X, where the shear layer in the wake of the upper stage interacts with the bow shock on the first stage, and results in an abrupt transition from a high drag to a low drag state of the shock wave. Another example of high gradients due to a bi-modal flow behavior is the abrupt wing stall issue that occurred on the pre-production F/A-18E. ${ }^{9}$ In this case, unsteady computations performed by Forsythe \& Woodson, ${ }^{10}$ in agreement with experiments, showed that the shock wave on top of the wing would dynamically oscillate between two stable positions at steady transonic conditions. This resulted in abrupt shock-induced flow separation on one side of the aircraft and large rolling moments that could not be controlled by the automatic flight control system.

The above phenomena can be present to varying degrees in all vehicle classes (commercial and military transport, rotorcraft, UAV, launch vehicles, capsules, etc), and throughout the speed regime. The techniques presented in this paper apply to all vehicle types that are subject to high gradient aerodynamics. The present work was developed in the context of launch abort system aerodynamics, therefore the examples used below pertain to blunt body type aerodynamics. Blunt bodies often present geometrical features that almost ensure high-gradient aerodynamic characteristics since they usually experience high angles of attack due to reorientation phases of flight, low dynamic and static stability margins, flight under drogue chutes and large parachutes, etc. As an example, the aerodynamic characteristics of a capsule, as it is pitched from low angles of attack (nose forward) to high angles of attack (heat shield forward) experiences a transition from fully attached flow on the windward walls of the capsule and massively separated flow on the leeward heat shield to a separating flow on the walls and reattachment of the flow on the heat shield. Roshko ${ }^{11}$ showed how sensitive bluff body aerodynamics are, in particular base suction and drag, to parameters like Reynolds number and the state of separation of the shear layer. This highly dynamic combination of aerodynamic phenomena results in very large gradients in the integrated forces and moments. Capturing this gradient with an appropriate uncertainty model is key.

\section{B. Physical Boundary Uncertainties}

The aerodynamic environment of a vehicle can be interpreted as a 'map' among physics-based inference spaces. From this perspective, there are two main challenges to be addressed: 1) Identifying the domain boundaries and the uncertainty in their characterization, and 2) Determining the aerodynamics of the vehicle and it's associated uncertainty within each domain. Wind tunnel measurements and computational simulations will provide an estimate of the boundaries of the physical domains and insight into the mechanisms that trigger the transition from one domain to another. It is however difficult to understand how well this boundary is determined due to the assumptions that were made when making the measurements at smaller scales, or the consequences of using a particular CFD model. Different turbulence models have strengths and weaknesses in predicting various types of flows, and phenomena like flow separation and boundary layer transition are always very challenging. Luckring et al. ${ }^{12}$ discuss how determining the boundaries of a physics-based inference space can allow for a validation of the computational code within a whole domain rather than at discrete locations, thereby providing the user an increased confidence in the simulations.

The work by Polhamus ${ }^{2}$ is an excellent example of how physical boundaries can be determined. Figure 1 is adapted from his work and shows the boundaries of different types of stall an airfoil would be subject to when varying Reynolds number and leading-edge radius. The boundaries of these physics-based inference 


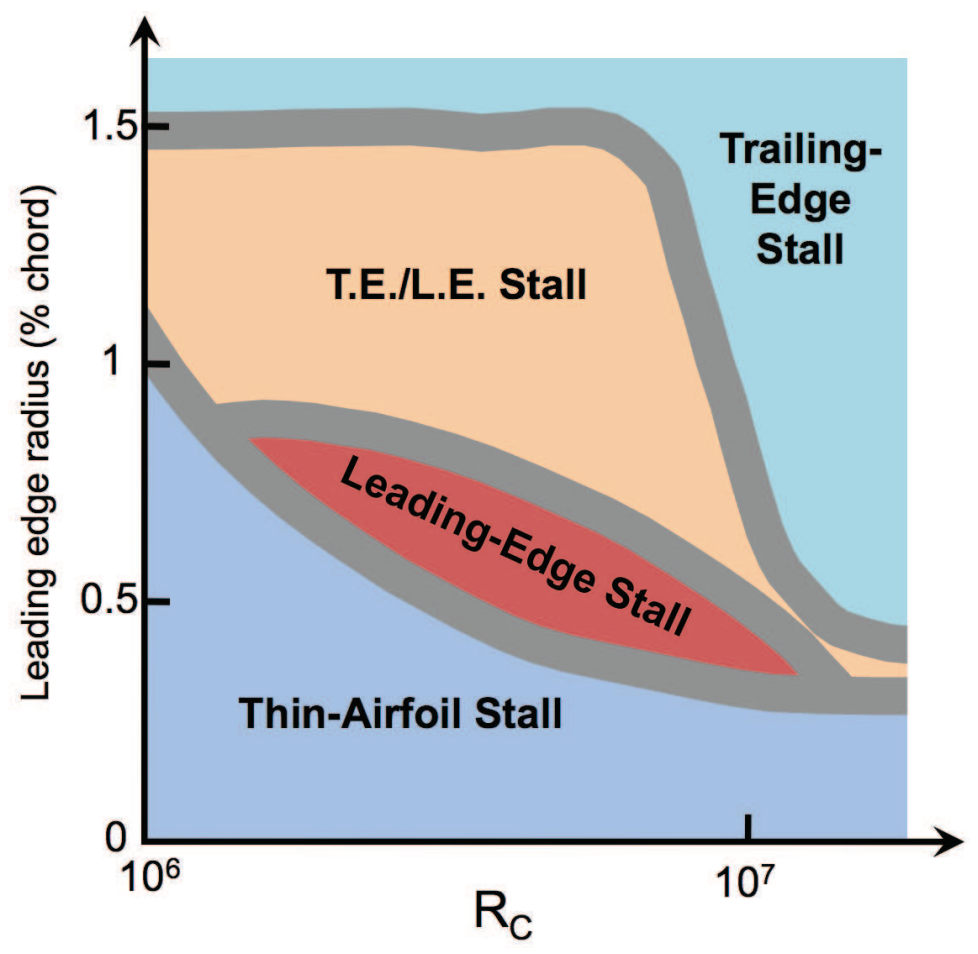

Figure 1. Stall domains for NACA 6-series symmetrical airfoils as a function of Reynolds number based on chord, $R_{C}$, and leading edge radius. Adapted from Polhamus. ${ }^{2}$

spaces having been determined, one of the challenges lies in quantifying the uncertainty in the location of these boundaries.

As described in the previous section, most forms of high-gradient phenomena are usually accompanied by strong unsteadiness. Capturing the magnitude of the unsteadiness and performing detailed measurements or computations in the vicinity of the transition between domains could provide useful information for quantifying how well the transition location is known. As an example, the dynamics of a shock wave interacting with a boundary layer at transonic conditions could result in the static location of a shock on an airfoil to become dynamic and the surface pressure distribution to exhibit a large discontinuity that travels with the shock. Performing unsteady pressure measurements with a dense row of sensors around the predicted shock location would provide for a more accurate measure of how the shock wave travels within a range of locations. This range could be used to determine uncertainty bounds for the location of the discontinuity in the pressure trace. It is shown in the next section how such a quantity can be used to model a phase uncertainty in a signal that exhibits a large gradient.

\section{An Asymmetric Uncertainty Expression for High-gradient Aerodynamics}

Uncertainty expressions are traditionally calculated from a dataset using a mix of engineering and statistical methods, which results in bounds that quantify the potential error in the output for a given input, i.e., a magnitude error or 'vertical' uncertainty. The article by Hemsch et al. ${ }^{13}$ describes in detail the process involved in the estimation of aerodynamic uncertainties for the Ares I launch vehicle during the ascent phase of flight. In most instances, when the data trends only exhibit small gradients, a pure magnitude uncertainty is largely sufficient to cover for potential phase errors, even though unaccounted for in the analysis. When gradients become large, magnitude-error uncertainties are no longer sufficient and a model that explicitly accounts for phase-error is needed. In the current paper, the uncertainty in the knowledge of the location of the abrupt physics changes will be referred to as a phase uncertainty since it is, in fact, an error in the output that is due to a lack of knowledge in the input, which can also be viewed as a 'horizontal' uncertainty. 


\section{A. Traditional Pure-magnitude Symmetric Uncertainty Expression}

Given an aerodynamic coefficient $C_{i}(X)$, the upper uncertainty bound $u^{+}(x)$, and the lower uncertainty bound $u^{-}(x)$ are defined as:

$$
\begin{aligned}
& u^{+}(x)=C_{i}(x)+\Delta u^{+} \\
& u^{-}(x)=C_{i}(x)-\Delta u^{-}
\end{aligned}
$$

Then, the traditional model for a constant symmetric uncertainty of magnitude $U$ is as follows:

$$
\begin{aligned}
& \Delta u^{+}(x)=U \\
& \Delta u^{-}(x)=U
\end{aligned}
$$

Figure 2 shows a lift-to-drag ratio polar for the Apollo command module as it is pitched from 0 to $90^{\circ}$ angle of attack, $0^{\circ}$ being apex pointed forward and $180^{\circ}$ being heat shield pointed forward. A strong gradient is seen in this data starting at approximately $65^{\circ}$ angle of attack, where the vehicle suddenly loses lift, indicating flow separation on the leeward curved walls of the command module and reattachment on the heat shield. A constant and symmetric uncertainty has been applied to the data, following established practices. In this case the magnitude of the uncertainty is arbitrary for the purpose of the demonstration. In regions where the data does not exhibit high gradients, the uncertainty model looks reasonable and would allow for errors in phase. However, when the gradients become very large, as in the region between 60 and $70^{\circ}$ angle of attack, this type of model unfortunately allows for only a very small range of phase error. In addition, this happens at a very critical condition during the flight, when the capsule reorients itself to present its heat shield forward for reentry and aerodynamic stability of the vehicle is key. Locally increasing the uncertainty magnitude (even grossly) would not accomplish the objective of allowing for phase error. Indeed, in the extreme case of a step function or an infinite local slope, even an infinitely large single-point uncertainty would not be satisfactory. Figure 3 shows a theoretical example of a step function (infinite gradient) with an arbitrary traditional constant symmetric uncertainty. It is clear that no phase-error is allowed with such a model in the high gradient area around $X=0.5$.

Such models imply perfect knowledge of the location of the high gradient or abrupt physical transition. What is needed is an uncertainty model for the condition of the abrupt transition from one domain to another. The following section describes a proposed model to fill this void.

\section{B. New Phase and Magnitude Asymmetric Uncertainty Expression}

The proposed expression for an asymmetric uncertainty that accounts for phase errors, given an estimated uncertainty in the knowledge of the location of the abrupt physics change, $\Delta x$, is as follows:

$$
\begin{aligned}
& \Delta u^{+}(x)=U+\left|C_{i}(x)-C_{i}\left(x+\Delta x \operatorname{sgn}\left(\frac{d C_{i}}{d x}\right)\right)\right| \\
& \Delta u^{-}(x)=U+\left|C_{i}(x)-C_{i}\left(x-\Delta x \operatorname{sgn}\left(\frac{d C_{i}}{d x}\right)\right)\right|
\end{aligned}
$$

where $\Delta u^{+}$is the positive uncertainty, $\Delta u^{-}$is the negative uncertainty, $U$ is the previously calculated singlepoint estimate of aerodynamic measurement uncertainty, and $C_{i}(x)$ is the aerodynamic coefficient at location $x$. This model simply allows for a phase-uncertainty in the input, $\Delta x$, to be propagated through the nominal data and provide an additional asymmetric uncertainty that is proportional to the gradient of the nominal function, and a function of the estimated input phase-uncertainty, $\Delta x$. If the gradient is positive, the upper uncertainty increment, $\Delta u^{+}(x)$, will spike slightly preceding the large gradient, and the lower uncertainty increment, $\Delta u^{-}(x)$, will spike slightly following the large gradient, increasing the 'width' of the uncertainty band, and effectively adding a phase-uncertainty at the location of the large gradient. The following example illustrates this feature. 

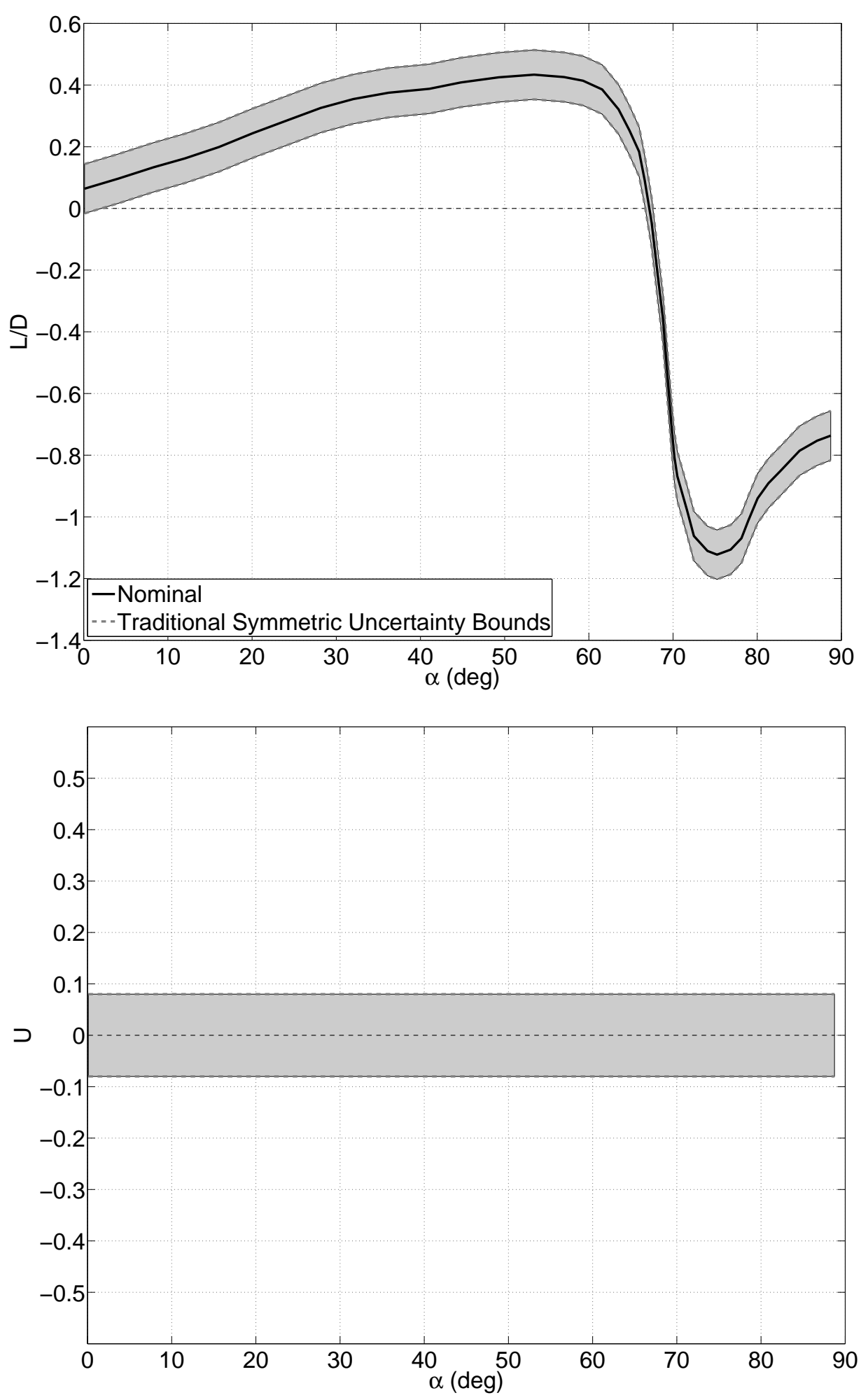

Figure 2. (top) Apollo command module lift-to-drag ratio at $M=0.7$, with traditional symmetric uncertainty bounds of arbitrary magnitude and (bottom) uncertainty bounds only. Lift-to-drag ratio data from Moseley et al. ${ }^{1}$ 

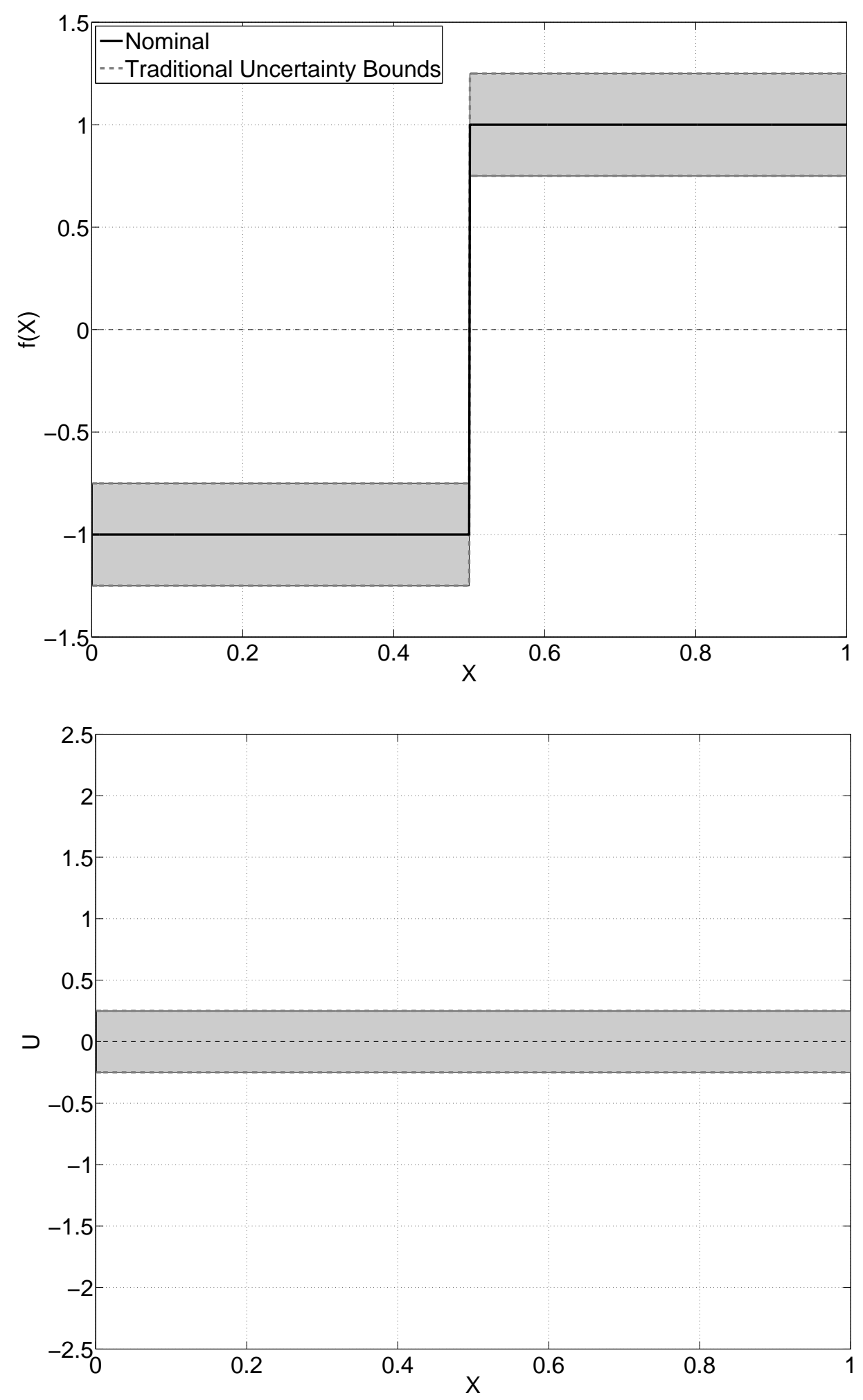

Figure 3. (top) Traditional uncertainty bound applied to a step function with arbitrary symmetric uncertainty, and (bottom) uncertainty bounds only. 
Using the Apollo command module example, the uncertainty model for lift-to-drag ratio as a function of angle of attack, $\alpha$, is as follows:

$$
\begin{aligned}
& \Delta u^{+}(\alpha)=U+\left|(L / D)(\alpha)-(L / D)\left(\alpha+\Delta \alpha \operatorname{sgn}\left(\frac{d(L / D)}{d \alpha}\right)\right)\right| \\
& \Delta u^{-}(\alpha)=U+\left|(L / D)(\alpha)-(L / D)\left(\alpha-\Delta \alpha \operatorname{sgn}\left(\frac{d(L / D)}{d \alpha}\right)\right)\right|
\end{aligned}
$$

Figure 4 shows the new uncertainty model applied to the Apollo command module lift-to-drag ratio. For the purpose of demonstrating the technique, a phase-uncertainty of $\Delta \alpha=1.5^{\circ}$ was arbitrarily chosen. The uncertainty band now allows for phase or 'horizontal' error in the high-gradient regions of the aerodynamics of the vehicle, without dramatically increasing the uncertainty magnitudes in the low-gradient regions. In areas of zero gradient, the added term vanishes leaving only the traditional single-point uncertainty estimate. Figure 5 shows both uncertainties together to illustrate this feature.

As applied to the theoretical step function, with an arbitrary uncertainty in the knowledge of the physical change of $\Delta X=0.025$, the new uncertainty model is shown in Fig. 6. When compared to Fig. 3, the addition of a phase-error now allows for modeling a variation in the flight simulations.

The phase-uncertainty, $\Delta x$, in the proposed model described by Eq. 3 has to be estimated using available aerodynamic data, as well as engineering judgment. Insight can be gained from testing the assumptions made when designing the experiment or the computational problem. For example, when testing a bluff body in a wind tunnel, Reynolds number could be a factor that greatly affects the nature of the flow separation, and in turn base pressure and longitudinal forces and moments. If the wind tunnel has the capability of varying Reynolds number at a fixed Mach number, and if time permits, one could perform such an investigation over a range of Reynolds numbers to get a sense of the sensitivity of the aerodynamic environment to Reynolds number. Testing at different scales and if possible in different tunnels can also provide valuable information in determining more accurately an uncertainty in the boundaries of the flow domains. Computationally, analyzing solutions from several turbulence models, grid characteristics, etc could result in variability in the predicted domain boundary and provide valuable information for an estimate of the phase-uncertainty.

As shown above, this new uncertainty model provides an effective mathematical way of propagating a phase-uncertainty through the aerodynamic data and results in uncertainty bounds that include both magnitude and phase uncertainty in the highest-gradient areas, where pure-magnitude uncertainty models do not. However this is not enough to ensure that, in a Monte-Carlo type dispersion analysis (as performed by Guidance, Navigation and Controls), every dispersion within the new uncertainty bounds will be realistic. A physics-based dispersion model, as presented in the following section, is recommended to introduce constrained and realistic randomness in the dispersed data.

\section{A Physical Dispersion Model for Asymmetric Uncertainty Expressions}

Traditionally, aerodynamic databases consist of: 1) a set of nominal coefficients that are a function of a number of independent variables, and 2) an uncertainty model that is usually a function of a subset of these variables. It was shown by Pinier ${ }^{14}$ that a physically meaningful dispersion model is highly beneficial to enable realistic modeling of the aerodynamic data within the flight simulations. The negative effects of an inappropriate dispersion model can have much more of an impact on vehicle performance than the actual uncertainty magnitudes. In cases where uncertainties about the nominal values are large, the absence of a physics-based dispersion model could lead to non-realistically dispersed data and artificially distorted simulation results that will not accurately represent the launch vehicle's behavior in flight. The Fourier series-based dispersion model introduced by Pinier ${ }^{14}$ and now implemented within NASA projects to more realistically represent random variations in flight simulations was developed for traditional symmetric single-

point uncertainty bounds. However this approach can be generalized to asymmetric uncertainty expressions that account for lack-of-knowledge type phase-errors, as described in the previous sections. 

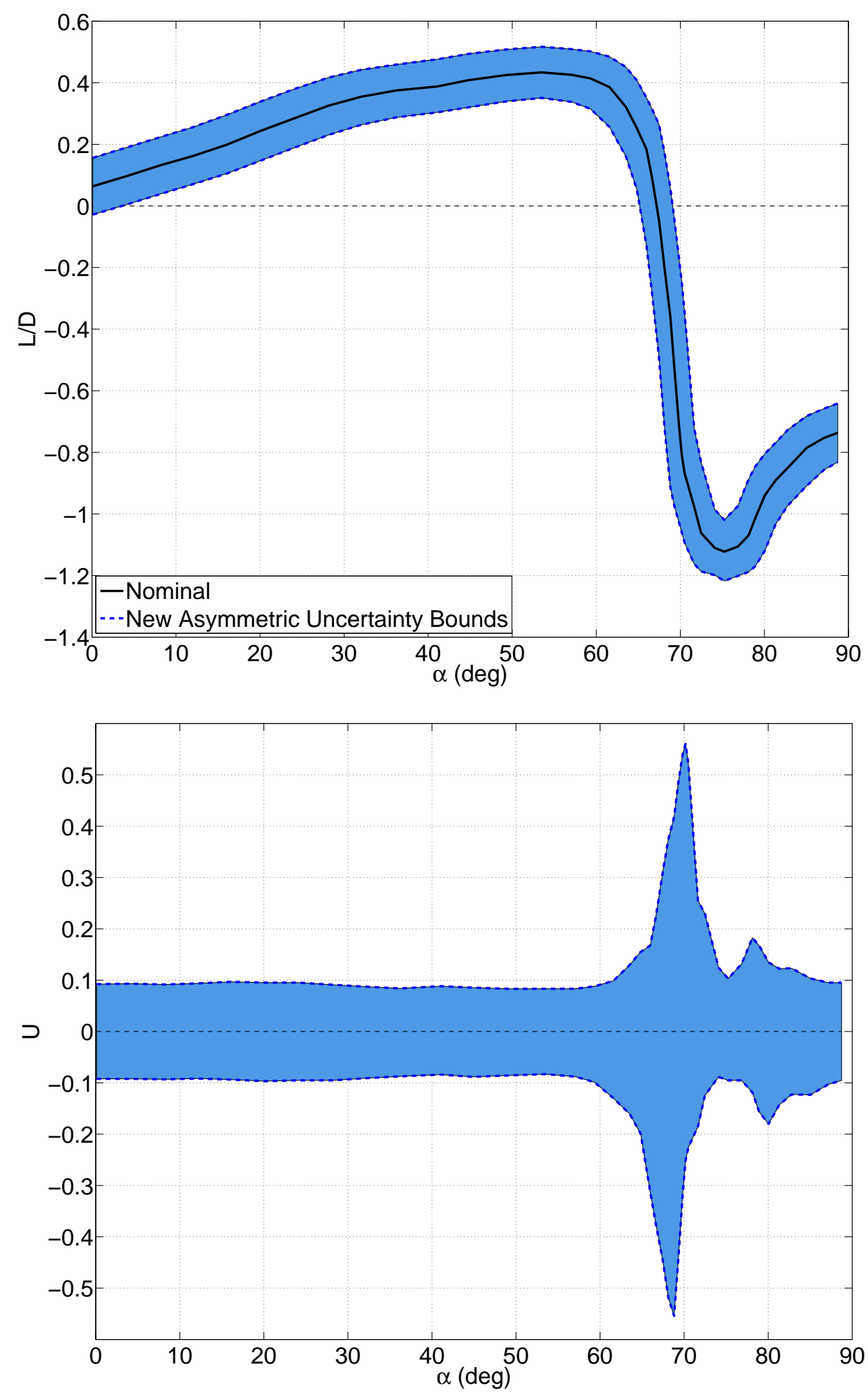

Figure 4. (top) Apollo command module lift-to-drag ratio at $\mathrm{M}=0.7$, with new asymmetric uncertainty bounds and (bottom) uncertainty bounds only. Lift-to-drag ratio data from Moseley et al. ${ }^{1}$ 


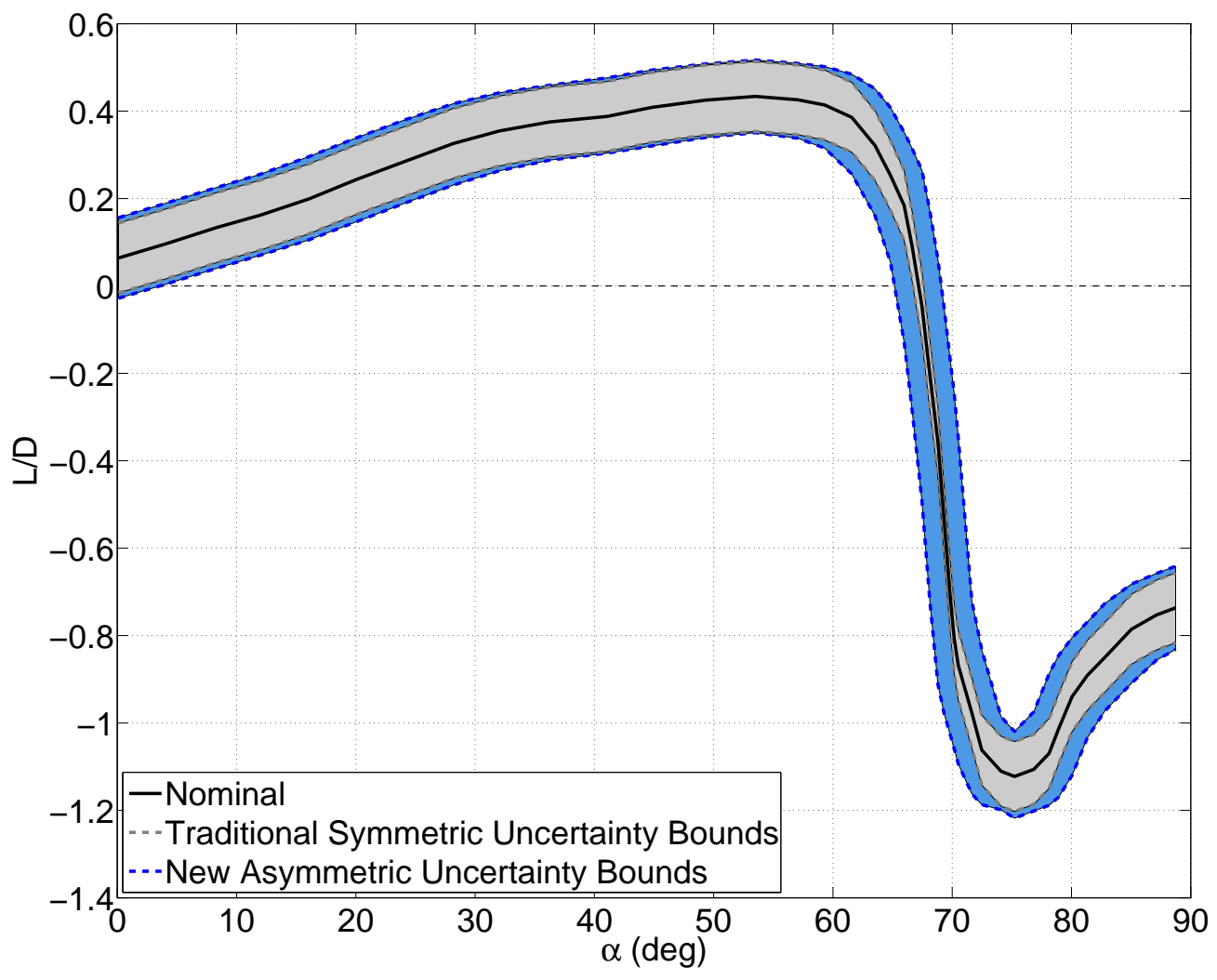

Figure 5. Apollo command module lift-to-drag ratio at $M=0.7$, with traditional symmetric uncertainty bounds (in gray) and new asymmetric uncertainty bounds (in blue). Lift-to-drag ratio data from Moseley et al. ${ }^{1}$ 

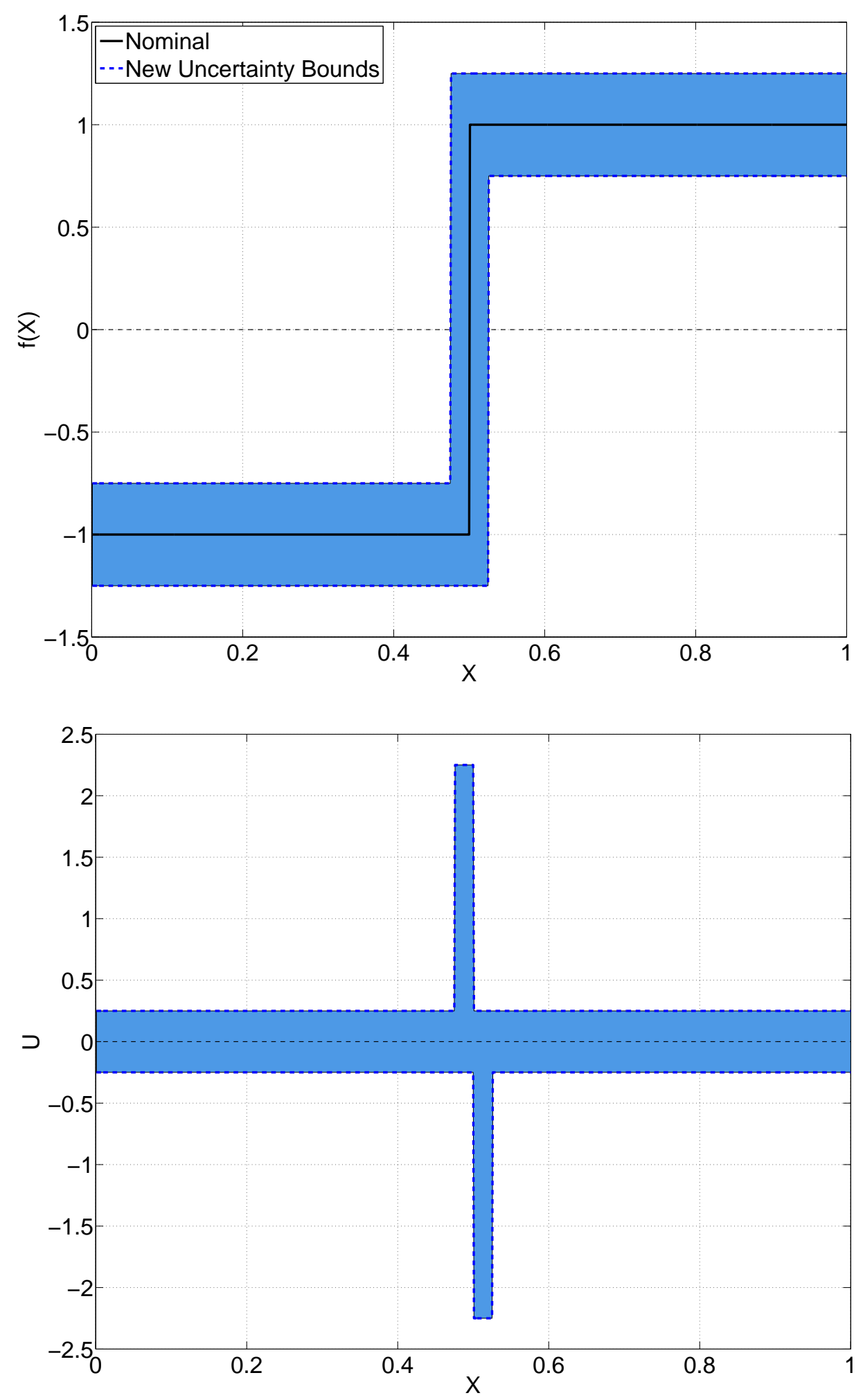

Figure 6. (top) New asymmetric uncertainty model applied to a step function with arbitrary uncertainty magnitude, and (bottom) new uncertainty bounds only. 


\section{A. Fourier Series based Dispersion Method as Applied to Symmetric Uncertainties}

Prediction data can differ from the truth in two ways: (1) bias error and (2) random error. It can also be a combination of the two. The model proposed by Pinier ${ }^{14}$ allows for both bias and random errors to be modeled at the same time in the dispersed data, while remaining constrained by the provided uncertainty bounds. The dispersion model consists of a traditional bias uncertainty term and an additional uncertainty term with a functional dispersion factor $g_{i}(x)$, as follows:

$$
\widetilde{f}(x)=f(x)+\epsilon_{i} L_{b} U(x)+\left(1-\left|\epsilon_{i} L_{b}\right|\right) g_{i}(x) U(x)
$$

where $\widetilde{f}(x)$ is the dispersed function. $U(x)$ is the uncertainty magnitude, $\epsilon_{i}$ is a randomly sampled uncertainty factor with $-1 \leq \epsilon_{i} \leq 1$. $L_{b}$ is the bias limit, with $-1 \leq L_{b} \leq 1$ and the dispersion function $g_{i}(x)$ is a randomly generated dispersion function that satisfies $-1 \leq g_{i}(x) \leq 1$. This dispersion model allows for the presence of a bias uncertainty limited to a magnitude of $L_{b} U(x)$, with the remaining uncertainty band allowed to be modeled as a pseudo-random function of $x$. The following partial sum of Fourier series is the proposed general model for the dispersion function $g_{i}(\bar{x})$ :

$$
g_{i}(\bar{x})=\sum_{k=1}^{N}\left(a_{k} \sin \left(2 \pi\left(k \bar{x}+h_{k}\right)\right)+b_{k} \cos \left(2 \pi\left(k \bar{x}+h_{k}\right)\right)\right)
$$

where $a_{k}, b_{k}$ and $h_{k}$ are randomly generated real numbers uniformly distributed between -1 and 1 , and $\bar{x}$ varies on a normalized scale from 0 to $1 . N$ is the order of the partial Fourier series. The $a_{k}$ and $b_{k}$ coefficients are the random magnitudes of the sines and cosines respectively and the $h_{k}$ coefficients are the random phases. The random phase enables the uncertainty band to be fully covered by the dispersed functions. Two parameters need to be chosen a priori to implement this model: the order of the Fourier series, $N$, and the bias limit, $L_{b}$. These parameters are determined using the following combination of physical reasoning and empirical data analysis:

1. The order of the partial Fourier series, $N$ : It is argued that any randomly dispersed function should exhibit a frequency content of the same order than that of the nominal function itself. Therefore the order of the series should not be higher than half the number of local extrema in the nominal function. As an example, the nominal function in Fig. 2 contains 2 local extrema and therefore the model should be of order $N=1$.

2. The bias limit, $L_{b}$ : In most cases, it is unrealistic to assume that $100 \%$ of the uncertainty could be due to systematic error and therefore be modeled by a simple bias. In some cases, a strong physical argument can be made to justify setting the bias limit to zero (as is the case in the Ares I rolling moment example provided in Pinier ${ }^{14}$ ). However, most of the time, a portion of the uncertainty can be attributed to systematic errors and the remaining portion attributed to randomized error sources. An empirical study of the available data (from flight tests, wind tunnel tests, and computational fluid dynamics) and an engineering argumentation to determine the potential sources of systematic and randomized errors are required to determine the magnitude of the bias limit $L_{b}$.

In addition, the dispersion function is constrained by: $\left|g_{i}(x)\right| \leq 1$, so that every dispersed function remains within the pre-defined uncertainty bounds. As shown in Eq. 6, this model does not satisfy this constraint. There are two ways to force all dispersed functions $g_{i}(x)$ to be smaller than 1 in absolute value. The reader is referred to the paper by Pinier ${ }^{14}$ for a detailed description of the method.

\section{B. Generalization of the Fourier Series based Dispersion Method to Asymmetric Uncertainty Expressions}

Section II showed how a rather simple model can result in more realistic uncertainty bounds for aerodynamic data that contains large gradients. These new uncertainty bounds allow for a phase-error in the data that is directly correlated to the amount of uncertainty in the knowledge of the location in time or space of the large gradient. The resulting uncertainty bounds, as shown in Fig. 6 are now largely asymmetric, i.e. the upper uncertainty increment $\Delta u^{+}$is not equal to the lower uncertainty increment $\Delta u^{-}$. 
Before the Fourier series based method was introduced by Pinier, ${ }^{14}$ the dispersion model that has been traditionally used applies a bias-only increment to the nominal data within the uncertainty bounds. The following equation describes a bias-only dispersed function for asymmetric uncertainty bounds:

$$
\widetilde{f}(x)=f(x)+\left(\frac{\Delta u^{+}(x)-\Delta u^{-}(x)}{2}\right)+\epsilon_{i}\left(\frac{\Delta u^{+}(x)+\Delta u^{-}(x)}{2}\right)
$$

Figure 7 shows an example for this type of bias-only dispersion method, as applied to the Apollo command module lift-to-drag ratio data and an asymmetric uncertainty band. It is clear that significant biases are introduced in every dispersed function and that more randomness would be expected to occur in a typical flight, given this data and its uncertainty band.

To disperse the nominal data within the asymmetric uncertainty bounds using the Fourier series based dispersion method, and to ensure that the entire uncertainty space can be covered, the data can be dispersed about the function that is equal to the average of the upper and lower uncertainty bounds: $\frac{\Delta u^{+}(x)-\Delta u^{-}(x)}{2}$. The following equation describes how the bias-only dispersion model from Eq. 7 is generalized to an asymmetric uncertainty model, as that proposed in this paper:

$$
\widetilde{f}(x)=f(x)+\left(\frac{\Delta u^{+}(x)-\Delta u^{-}(x)}{2}\right)+\left(\epsilon_{i} L_{b}+\left(1-\left|\epsilon_{i} L_{b}\right|\right) g_{i}(x)\right)\left(\frac{\Delta u^{+}(x)+\Delta u^{-}(x)}{2}\right)
$$

In the particular case where the uncertainty is symmetric, $\Delta u^{+}(x)=\Delta u^{-}(x)=U(x)$, and this equation is equivalent to the traditional model from Eq. 5.

Figure 8 shows an example for this Fourier series based dispersion method, as applied to the Apollo command module lift-to-drag ratio data and an asymmetric uncertainty band. The bias limit $L_{b}$ in this case is equal to zero for a clearer representation of the added randomness from the Fourier series based method. The resulting dispersed functions exhibit more random magnitude, slope and phase variations, which allows for the flight simulation to be stressed in more ways than a pure bias type dispersion method.

\section{Conclusion}

In aerodynamic uncertainty quantification high-gradient areas are traditionally treated in the same manner as low-gradient areas, with mostly statistically-derived symmetric bounds about a nominal function. The inherent limitation of such techniques is that the phase or 'horizontal' uncertainty in the high-gradient areas becomes unrealistically small. The present work has introduced a general mathematical model that results in an asymmetric uncertainty, the magnitude of which is proportional to the gradient in the nominal data. This allows for phase-error in high-gradient aerodynamic regions, where traditional pure-magnitude symmetric uncertainty models do not. Within a physical domain, where gradients are generally low, the size of the additional term becomes insignificant. Additionally, a generalization of the randomized Fourier-series based dispersion model extends the author's previous work to allow for more realistic Monte-Carlo type dispersions to be combined with the proposed asymmetric uncertainty bounds, and results in the inclusion of a phase dispersion in the predictive data around physical domain transitions.

The combination of a high gradient uncertainty model, and a more randomized physics-based dispersion method, as proposed in this paper, provides for more realistic estimates of uncertainty in applications where phenomena such as flow separation/reattachment, shockwave/boundary layer interactions, laminar-turbulent transition, or bi-modal dynamics play a key role in the aerodynamic characteristics of the vehicle. It is argued that aerodynamic uncertainty expressions that contain physics-based models are more representative of the full scale flight vehicle in the flight simulations, which results in a more robust evaluation of the flight risks and, in turn, an increased probability of success. 

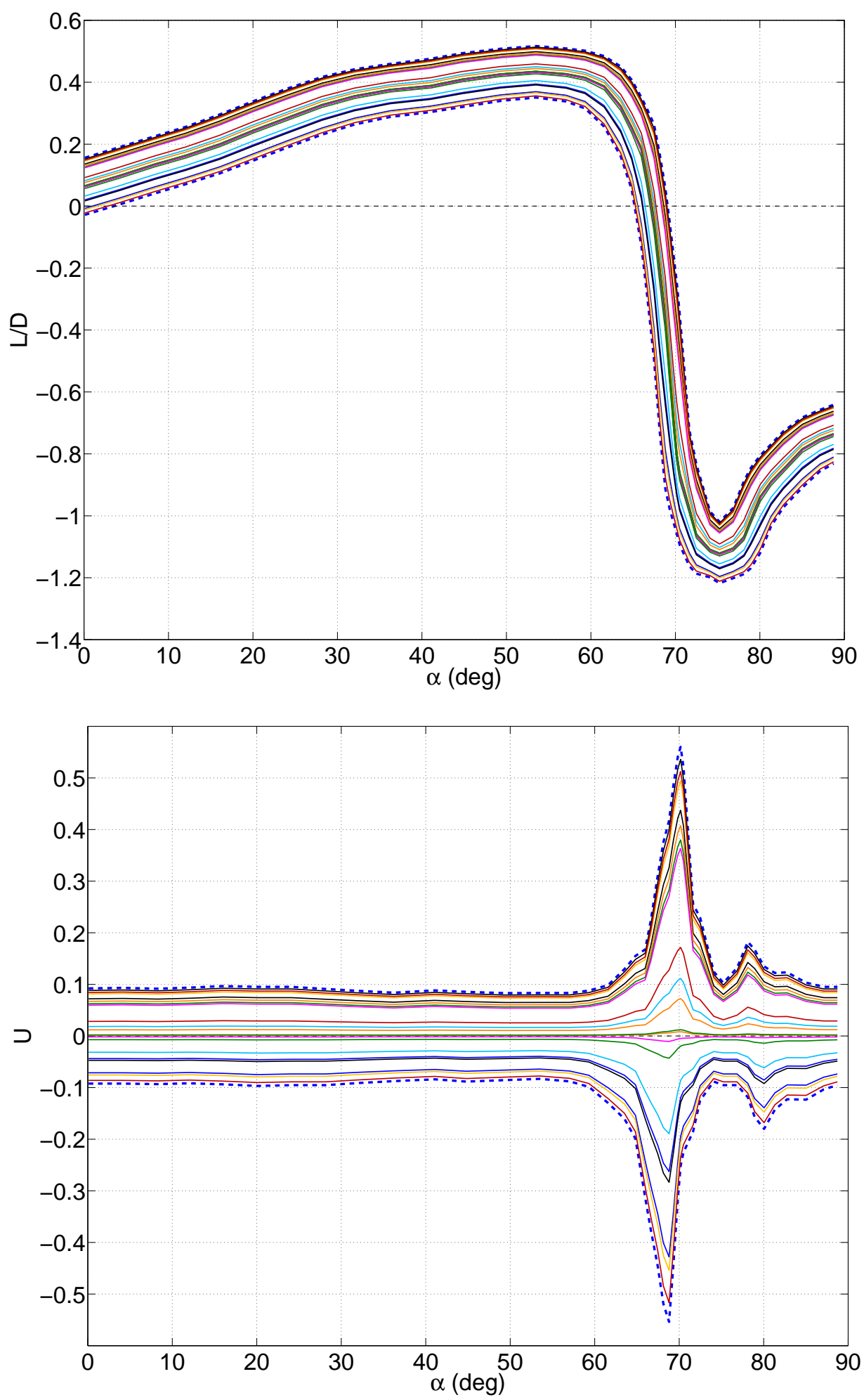

Figure 7. (top) Apollo command module lift-to-drag ratio at $M=0.7$, with new asymmetric uncertainty bounds and 20 bias-only dispersions, and (bottom) asymmetric uncertainty bounds and bias-only dispersion functions. Lift-to-drag ratio data from Moseley et al. ${ }^{1}$ 

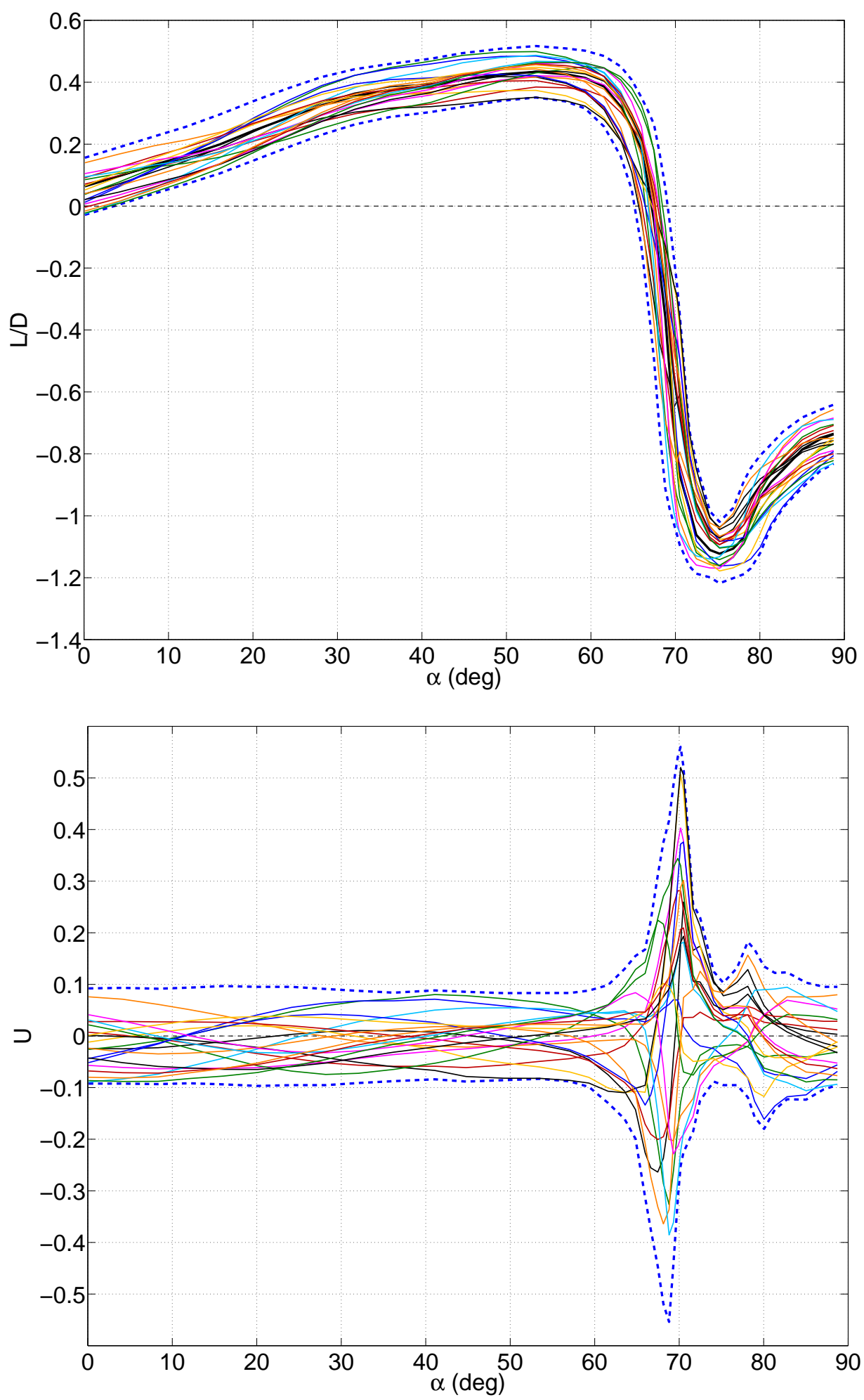

Figure 8. (top) Apollo command module lift-to-drag ratio at $M=0.7$, with new asymmetric uncertainty bounds and 20 Fourier series based dispersions, and (bottom) asymmetric uncertainty bounds and Fourier series based dispersion functions. Lift-to-drag ratio data from Moseley et al. ${ }^{1}$ 


\section{Acknowledgements}

The author would like to acknowledge the Launch Abort System Risk Mitigation Project and the NASA Engineering and Safety Center (NESC) for supporting this research, the goal of which has been to improve our capabilities for assessing risk associated with the aerodynamics of launch vehicles and launch abort systems, by developing more physically realistic uncertainty models and dispersion methodologies. Discussions with Drs. Hemsch, Luckring and Walker from NASA LaRC have also provided great insight into better understanding how these methods could be beneficial to the field of aerodynamic uncertainty quantification.

\section{References}

\footnotetext{
${ }^{1}$ Moseley, W. C., Graham, R. E., and Hughes, J. E., "Aerodynamic Stability Characteristics of the Apollo Command Module," NASA Technical Note, NASA TN D-4688, 1968.

${ }^{2}$ Polhamus, E. C., "A Survey of Reynolds Number and Wing Geometry Effects on Lift Characteristics in the Low Speed Stall Region," NASA CR-4745, 1996.

${ }^{3}$ Luckring, J. M., "A Survey of Factors Affecting Blunt Leading-Edge Separation for Swept and Semi-Slender Wings," 28th AIAA Applied Aerodynamics Conference, AIAA 2010-4820, 2010.

${ }^{4}$ Smits, A. J. and Muck, K.-C., "Experimental study of three shock wave/turbulent boundary layer interactions," Journal of Fluid Mechanics, Vol. 182, 1987, pp. 291-314.

${ }^{5}$ Braslow, A. L., Hicks, R. M., and Harris, R. V., "Use of grit-type boundary-layer-transition trips on wind-tunnel models," NASA TN D-3579, 1966.

${ }^{6}$ Blackwell, J. A., "Preliminary study of effects of Reynolds number and boundary-layer transition location on shockinduced separation," NASA TN D-5003, 1969.

${ }^{7}$ Piatak, D., Sekula, M., and Rausch, R., "Comparison of Ares I-X Wind-Tunnel Derived Buffet Environment with Flight Data," 29th AIAA Applied Aerodynamics Conference, AIAA-2011-3013, 2011.

${ }^{8}$ Pinier, J. T. and Niskey, C. J., "Ares I and Ares I-X Stage Separation Aerodynamic Testing," 49th AIAA Aerospace Sciences Meeting Including the New Horizons Forum and Aerospace Exposition, AIAA 2011-0169, 2011.

${ }^{9}$ Hall, R. M. and Woodson, S. H., "Introduction to the Abrupt Wing Stall Program," Journal of Aircraft, Vol. 41, No. 3, 2004, pp. 425-435.

${ }^{10}$ Forsythe, J. R. and Woodson, S. H., "Unsteady Computations of Abrupt Wing Stall Using Detached-Eddy Simulation," Journal of Aircraft, Vol. 42, No. 3, 2005, pp. 606-616.

${ }^{11}$ Roshko, A., "Perspectives on bluff body aerodynamics," Journal of Wind Engineering and Industrial Engineering, Vol. 49, 1993, pp. 79-100.

${ }^{12}$ Luckring, J. M., Hemsch, M. J., and Morrison, J. H., "Uncertainty in computational aerodynamics," 41st AIAA Aerospace Sciences Meeting and Exhibit, AIAA 2003-0409, 2003.

${ }^{13}$ Hemsch, M. J., Hanke, J. L., Walker, E. L., and Houlden, H. P., "Detailed Uncertainty Analysis for Ares I Ascent Aerodynamics Wind Tunnel Database," 26th AIAA Aerodynamic Measurement Technology and Ground Testing Conference, AIAA 2008-4259, 2008.

${ }^{14}$ Pinier, J. T., "A New Aerodynamic Data Dispersion Method for Launch Vehicle Design," 29th AIAA Applied Aerodynamics Conference, AIAA 2011-3167, 2011.
} 